\title{
The Application of Three-Dimensional Printed Finger Splints for Post Hand Burn Patients: A Case Series Investigation
}

\author{
Ho-Sung Nam, $\mathrm{MD}^{1}$, Cheong Hoon Seo, $\mathrm{MD}^{2}$, So-Young Joo, $\mathrm{MD}^{2}$, \\ Dong Hyun Kim, MD, $\mathrm{PhD}^{1}$, Dong-Sik Park, $\mathrm{MD}, \mathrm{PhD}^{1}$ \\ ${ }^{1}$ Department of Rehabilitation Medicine, Kangdong Sacred Heart Hospital, Seoul; \\ ${ }^{2}$ Department of Rehabilitation Medicine, Hallym University Hangang Sacred Heart Hospital, Seoul, Korea
}

The application of three-dimensional (3D) printing is growing explosively in the medical field, and is especially widespread in the clinical use of fabricating upper limb orthosis and prosthesis. Advantages of 3D-printed orthosis compared to conventional ones include its lower cost, easier modification, and faster fabrication. Hands are the most common body parts involved with burn victims and one of the main complications of hand burns are finger joint contractures. Applying orthotic devices such as finger splints are a well-established essential element of burn care. In spite of the rapid evolution of the clinical use of 3D printing, to our knowledge, its application to hand burn patients has not yet been reported. In this study, the authors present a series of patients with hand burn injuries whose orthotic needs were fulfilled with the application of 3D-printed finger splints.

Keywords Three-dimensional printing, Burns, Orthotic devices

\section{INTRODUCTION}

Hands are the most common body part injured when burns occur, although they comprise only $3 \%-5 \%$ of body surface area [1]. Post-burn patients suffer from various complications such as joint contracture, hypertrophic scars, and peripheral nerve injury. In particular, finger joints are the most common site of joint motion disorder [2]. Post-burn deformities of the hand are functionally limiting and serve as a visual stigma to the patient [3]. Comprehensive interventions including early excision and grafting, early joint mobilization, scar care, and splinting are necessary to help prevent joint contracture. Orthosis application is an important practice that should be maintained throughout the entire process of burn care. One domestic survey revealed finger splints were the most common prescription for burn patients after injury (44.2\%), and finger extension splints were the

Received July 26, 2017; Accepted October 10, 2017

Corresponding author: Dong Hyun Kim

Department of Rehabilitation Medicine, Kangdong Sacred Heart Hospital, 445, Gil-dong, Gangdong-gu, Seoul 05355, Korea. Tel: +82-2-2225-2789, Fax: +82-2-473-8101, E-mail: skyler02@hallym.or.kr

ORCID: Ho-Sung Nam (http://orcid.org/0000-0003-3188-2053); Cheong Hoon Seo (http://orcid.org/0000-0003-4091-6382); So-Young Joo (http:// orcid.org/0000-0002-1199-7278); Dong Hyun Kim (http://orcid.org/0000-0002-3102-385X); Dong-Sik Park (http://orcid.org/0000-0002-8977-7099).

(c) This is an open-access article distributed under the terms of the Creative Commons Attribution Non-Commercial License (http://creativecommons.org/ licenses/by-nc/4.0) which permits unrestricted noncommercial use, distribution, and reproduction in any medium, provided the original work is properly cited. Copyright $\odot 2018$ by Korean Academy of Rehabilitation Medicine 
most prevalent type of finger splints (29.3\%), followed by thumb web spacers [4].

Post-burn patients often require serial splinting to prevent grafted skin breakdown or a hypertrophic scar, and the majority of the orthosis should be fabricated individually to meet the special conditions of burn patients [4]. Although conventional orthoses made with thermoplastic techniques are most widely used, their complexity and higher cost can be a burden to burn patients. Patients' orthotic and prosthetic needs may be better met with simpler solutions. Along with the developments in computer aided design (CAD) programs, online open-source file sharing, and increasing availability of three-dimensional (3D) printers, there has been an increase in interest of the low-cost custom 3D printed orthotic devices. The authors report three cases of patients with deep hand burns whose post-burn joint contractures were successfully managed with the use of 3D-printed finger splints.

\section{CASE REPORTS}

CAD programs and 3D printers (Fig. 1) were used to fabricate the finger splints. Using the free open-source hand design, Thingiverse (MakerBot Industries, Brooklyn, NY, USA), all designs were modified individually with Rhinoceros 5.0 (Robert McNeel \& Associates, Seattle, WA, USA) or Simplify3D (Simplify3D, Cincinnati, OH, USA). Since the finger splint designs used in this study did not require total contact with the affected finger, we measured each patient's finger length with the small joints and modified the design with the above mentioned CAD programs instead of using a 3D scanner. The designed splint was printed with FlashForge Creator Pro (Flash-
Forge, City of industry, CA, USA) using the fused deposition modeling (FDM), which involved stacking up melted flexible thermoplastic elastomer filaments layer by layer [5]. The plastic monofilaments used were either thermoplastic polyurethanes (TPU) for the soft material splint or polylactic acid (PLA) for the hard material splint, which are the same thermoplastic materials used in manufacturing conventional orthosis. For post-processing, the rough surface of the $3 \mathrm{D}$ printed finger splints was smoothed by chisel and sandpaper.

\section{Case 1}

A 21-year-old female visited the ER due to bare skin defect on face, trunk, genitalia, and both upper and lower extremities. Deep second- and third-degree burns covering $47 \%$ of the whole body surface was diagnosed. Multiple surgeries including an escharectomy and allograft, and split-thickness skin grafts (STSG) were performed. When referred to the rehabilitation department, she showed left hand, shoulder, and right knee joint contractures. On physical exam, she showed distal interphalangeal joint (DIP), proximal interphalangeal joint (PIP) flexion, extension limitation, and angulation deformity in all digits of the left hand (Fig. 2). Active range of motion (ROM) of the left metacarpophalangeal (MP), PIP, and DIP joints was fixed to $0^{\circ}$. To prevent flexion contracture of finger joints and angulation deformity due to hypertrophic scaring, a 3D-printed hard material finger extension splint with PLA monofilaments was applied to her 2nd and 5th fingers. She was advised to keep the splints on for 24 hours a day, and other rehabilitation management included stretching of all contracted joints, wearing a compression garment, applying moisturizing treatments,
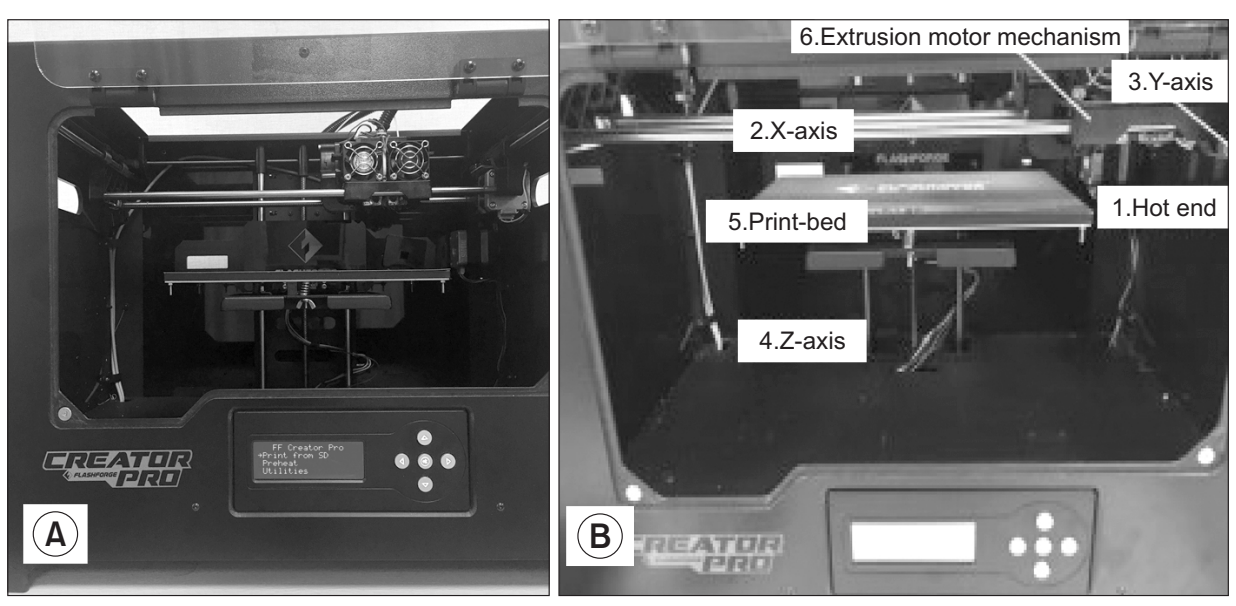

Fig. 1. (A) Three-dimensional (3D) printer (Creator Pro; FlashForge, City of industry, CA, USA). (B) Anatomy of the $3 \mathrm{D}$ printer. 

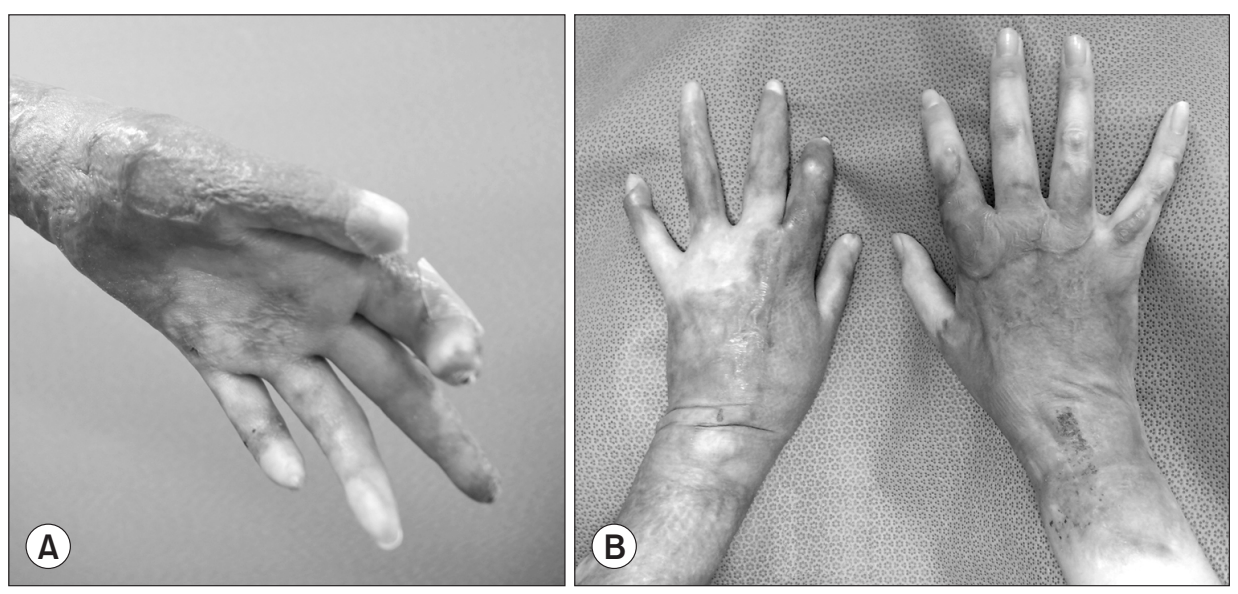

Fig. 2. Post burn hand deformity of Case 1. (A) All digits of the left hand and (B) both hands. followed by laser therapy for hypertrophic scar tissue. On the last follow-up evaluation after 18 months, her finger motion was sustained to avoid further reconstruction surgery for the hand deformity. Although she was not able to perform pinch and opposition with left fingers, the 3rd to the 5th digit MP, PIP, DIP ROM was improved to $30^{\circ}-40^{\circ}$ of flexion with full active finger extensions, and she could make a weak grasp. Total modified Barthel index (MBI) score was changed from 84 to 91 with the subsequent improvement of personal hygiene and in the bathing, feeding, and dressing domains.

\section{Case 2}

A 39-year-old female was referred to the rehabilitation outpatient clinic due to left 3rd to 5th finger contractures. Her left hand was crushed under a hot press machine, and she received early escharectomy, debridement and STSG for the bare skin defect on the left hand dorsum and 3rd to 5th fingers 3 months ago. On surgical exploration, her extensor tendon injury was extensive with 3rd to 4th PIP joint dislocations. She presented claw hand deformity with the 3rd, 4th, and 5th fingers MP extension and PIP flexion contracture (Fig. 3A, 3B). On initial ROM evaluation the 3rd, 4rth, and 5th MP flexion was limited to $70^{\circ}, 40^{\circ}, 55^{\circ}$, and PIP was fixed to $65^{\circ}, 60^{\circ}, 0^{\circ}$ of flexion, respectively. Electrodiagnostic evaluation revealed reduced amplitude of dorsal ulnar cutaneous nerve and abnormal spontaneous activities as well as reduced recruitment at dorsal interossei muscles, which suggests an ulnar nerve lesion at or around the burn site level. Since reconstruction surgery was not possible due to severe soft tissue defects, we applied a hard-material correction finger extension splint 3D printed with PLA monofilaments to her 3rd and 4th fingers to prevent flexion contracture (Fig. 3C, 3D). At examination 18 months later, ROM was slightly improved to 3 rd to 5 th MP flexion $85^{\circ}, 75^{\circ}, 65^{\circ}$; PIP flexion $80^{\circ}, 70^{\circ}, 0^{\circ}$; and PIP extension $-50^{\circ},-65^{\circ}, 0^{\circ}$, respectively. She was able to make a fist, perform all pinch motions and oppositions with all five fingers. Total MBI score remained constant with 95 requiring minimal assistance for bathing, feeding, and dressing domains.

\section{Case 3}

A 37-year-old male burned both of his hands while touching the peeled sheath of a cable. He was diagnosed with deep second- to third-degree electrical burns involving both of his hands, and received multiple surgeries including debridement and peroneal artery perforator free flap on the right 2 nd finger, and debridement and STSG of the right 3rd and 4th, and left 2nd fingers. Operative findings revealed necrotic change on the right 2nd extensor tendon, which was removed. When he was referred to the rehabilitation department, he presented a mallet finger deformity of the right 2nd finger (Fig. 4A). On ROM evaluation, the right 2nd finger DIP joint was fixed to $45^{\circ}$. At first, we applied a static molded plastic splint for the mallet finger (Fig. 4B). However, he showed poor compliance due to an irritation of the flapped dorsal skin underneath the splint. We changed the splint to the 3D-printed soft material finger extension splint with TPU monofilaments to minimize the skin irritation (Fig. 4C, $4 \mathrm{D})$. Due to good adherence to the new splint, his digit maintained a neutral position. Twelve months after the initial injury, he received flap debulking and local flap surgery to repair the extensor tendon gap of the right 2 nd finger. 

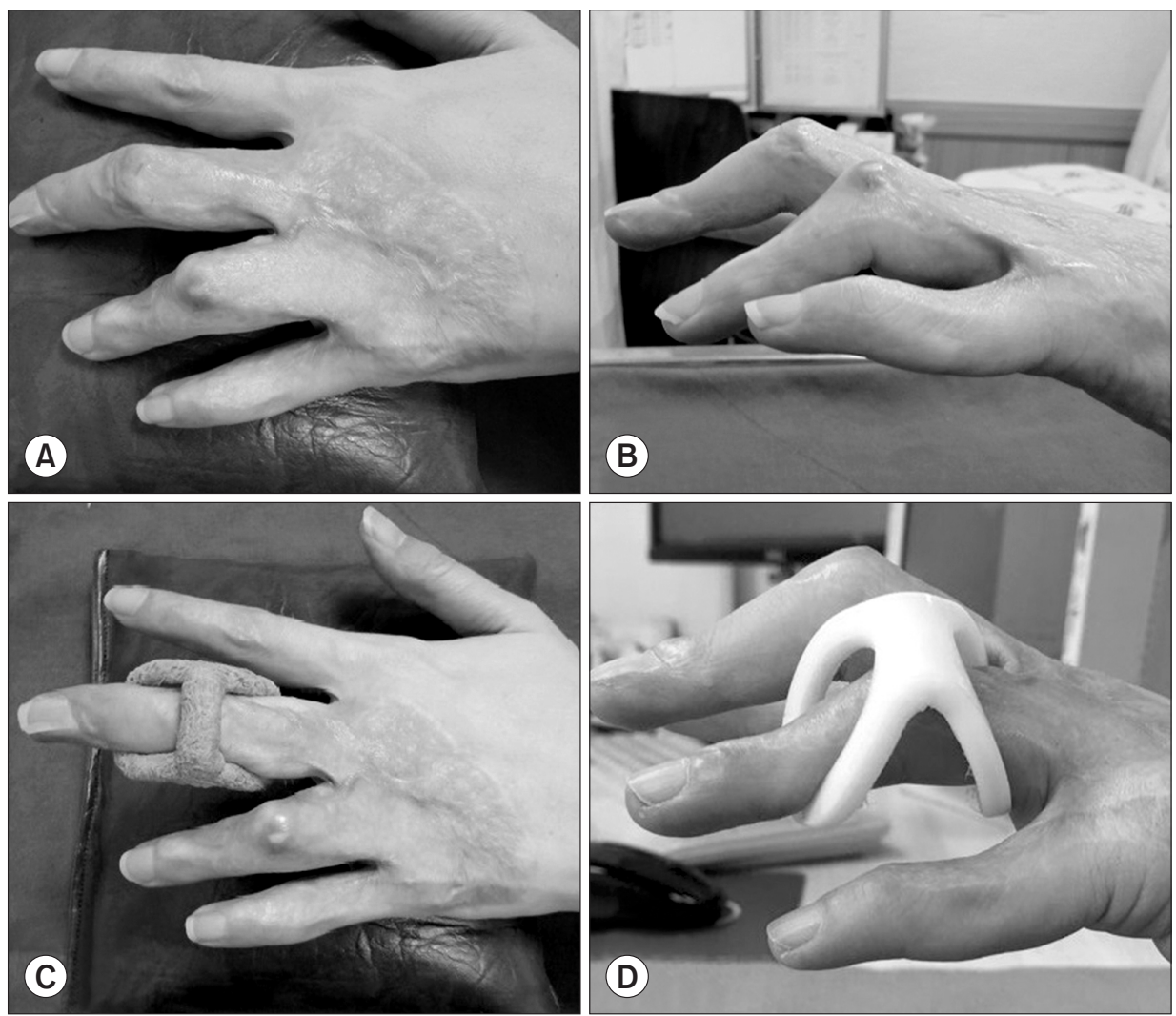

Fig. 3. (A, B) Post burn claw hand deformity caused by ulnar nerve injury. (C, D) Three-dimensional printed hard material finger extension splint design in Case 2.
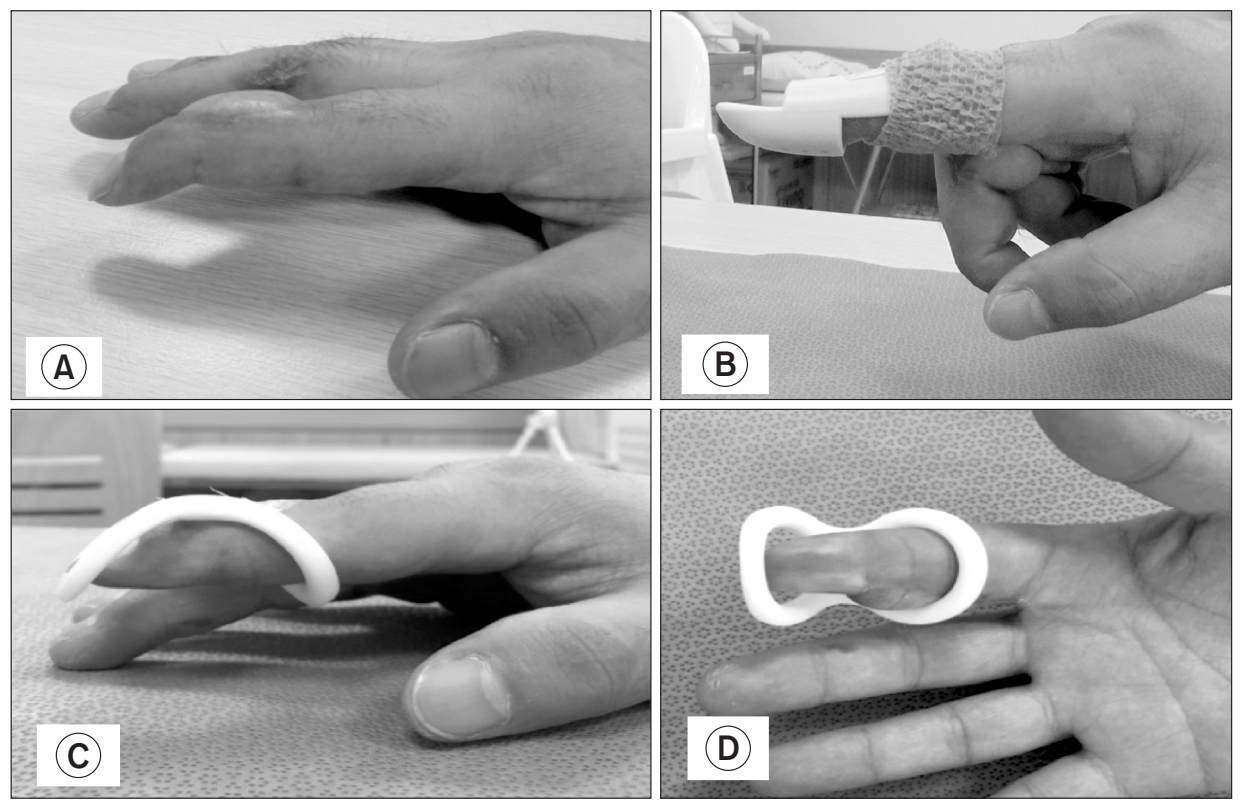

Fig. 4. (A) Post burn mallet finger deformity caused by extensor tendon injury. (B) Conventional plastic static splint. (C, D) Threedimensional printed soft material finger extension splint design in Case 3.

\section{DISCUSSION}

Along with the rapid evolution of CAD, 3D scanning, and capabilities of the 3D printer, the 3D printer's application to rehabilitation field bears tremendous po- tential. The availability of 3D printing offers the ability to fabricate low-cost, light-weight, durable, and easily replaceable hand and finger orthotic devices compared to preparing a conventional orthosis [6]. The greatest advantage to post-burn patients is its easy adjustability to fit 
the changing needs or underlying hand conditions using the stored 3D image files.

Compliance in using an orthosis has a very important role in improving the function of a patient. Although no systematic survey has been conducted on the adherence to the orthosis among the post-burn patients, noncompliance due to pain, edema, or skin problems are wellknown precautions preventing use [4]. Cost is another obstacle for the post-burn patients who often require serial changes of orthotic devices. In our three case investigations, one patient showed poor compliance to the conventional finger splint, and one patient hesitated to use the conventional orthosis due to the high cost. It took about 1 hour and less than US\$1 (considering only the cost of plastic monofilaments materials) to fabricate each finger splint. And all patients were satisfied with the use of $3 \mathrm{D}$ printed splints.

In this study, we applied finger extension splints to three patients made with CAD and a $3 \mathrm{D}$ printer. This is only an introductory report on the use of 3D printed splints in post-burn patients and it lacks a direct comparison between the conventionally applied splints. Another limitation of this study is that objective measures of patient satisfaction or functional outcome were not used. Further research should establish the feasibility of this technology as it might be a greater alternative to the conventional orthosis for post-burn patients.

\section{CONFLICT OF INTEREST}

No potential conflict of interest relevant to this article was reported.

\section{ACKNOWLEDGMENTS}

This research was supported by a grant of the Korea Health Technology R\&D Project through the Korea Health Industry Development Institute, funded by the Ministry of Health \& Welfare, Republic of Korea (No. HI15C1486).

\section{REFERENCES}

1. Sorkin M, Cholok D, Levi B. Scar Management of the Burned Hand. Hand Clin 2017;33:305-15.

2. Choi JS, Mun JH, Lee JY, Jeon JH, Jung YJ, Seo CH, et al. Effects of modified dynamic metacarpophalangeal joint flexion orthoses after hand burn. Ann Rehabil Med 2011;35:880-6.

3. Brown M, Chung KC. Postburn contractures of the hand. Hand Clin 2017;33:317-31.

4. Jang KU, Seo CH, Kim MK, Moon CH, Yang JI, Kim JW, et al. The distribution analysis of orthotics in burn rehabilitation. J Korean Burn Soc 2004;7:98-103.

5. Lee KH, Kim SJ, Cha YH, Kim JL, Kim DK, Kim SJ. Three-dimensional printed prosthesis demonstrates functional improvement in a patient with an amputated thumb: a technical note. Prosthet Orthot Int 2018;42:107-11.

6. Burn MB, Ta A, Gogola GR. Three-dimensional printing of prosthetic hands for children. J Hand Surg Am 2016;41:e103-9. 